[7] K. G. Zhao, J. Blabla, and J. Helmcke, " ${ }^{127} \mathrm{I}_{2}$ stabilized $\mathrm{He}^{22} \mathrm{Ne}$ laser at $640 \mathrm{~nm}$ wavelength," pp. 252-256, this issue.

[8] F. Bayer-Helms and J. Helmcke, "Modulation broadening of spectral profiles," PTB Bericht, Me-17, pp. 85-109, 1977.

[9] F. Spieweck, "Influence of small impurities in absorption cells of $\mathrm{I}_{2}$-stabilized lasers upon their frequency," pp. 246-248, this issue.

[10] P. Bertinetto, B. I. Rebaglia, P. Cordiale, S. Fontana, and G. B. Picotto, "He-Ne $\left({ }^{127} \mathrm{I}_{2}\right.$ ) lasers at $0.633 \mu \mathrm{m}$ (and at $\left.0.640 \mu \mathrm{m}\right)$," in Precision Meas. and Fundamental Constants II, NBS Spec. Publ. 617, pp. 69-72, 1984.

[11] W. R. C. Rowley, "Power shift of a $633-\mathrm{nm}{ }^{127} \mathrm{I}_{2}$ stabilized He-Ne laser, for various mirror curvatures," Nat. Phys. Lab., Teddington, England, 1981, NPL Rep. MOM 56.

[12] D. N. Ghosh Roy, F. Bertinetto, B. I. Rebaglia, and P. C. Cresto, "Effects of iodine saturation dispersion on the ${ }^{127} I_{2}$ stabilized He-Ne laser," J. Appl. Phys., vol. 54, pp. 531-534, 1983.

\title{
Laser Interferometric Sensing Techniques for Very Small Displacements - With Applications to Gravitational Radiation Detectors
}

\author{
H. WARD, J. HOUGH, G. P. NEWTON, B. J. MEERS, N. A. ROBERTSON, S. HOGGAN, G. A. KERR \\ J. B. MANGAN, AND R. W. P. DREVER
}

\begin{abstract}
In this paper we discuss the use of laser interferometry with resonant optical cavities to sense very small displacements of suspended test masses in a gravitational radiation detector. In particular we describe and discuss the performance of a prototype interferometer currently under development, which has high finesse cavities, 10-m long, and is illuminated by highly frequency-stabilized light from an argon laser. A displacement sensitivity close to $10^{-16} \mathrm{~m} / \sqrt{\mathrm{Hz}}$ at $\mathrm{kHz}$ frequencies, limited by photon noise in the detected light, is achieved.
\end{abstract}

\section{INTRODUCTION}

$A^{N}$ N AREA of measurement technology currently under development is the application of laser interferometry in the search for gravitational radiation from collapsing stars and other violent astronomical events.

Detection techniques' rely on sensing the minute changes $\mathrm{dl}$, in the separation of masses separated by a distance, 1 , and recent estimates of source strengths indicate that at frequencies near $1 \mathrm{kHz}$, probably the most promising region of the spectrum for initial ground-based experiments, a strain sensitivity, $\mathrm{d} 1 / 1$ of $10^{-21}$ or better should be aimed for.

A possible method of attaining the very high sensitivity required may be to use optical techniques to sense changes in the spacing of nearly free test masses separated by relatively large distances, perhaps up to a few kilometers [1]. A displacement sensitivity of the order of $10^{-18} \mathrm{~m}$ over a millisecond timescale is then required. To avoid absolute length

Manuscript received August 21, 1984. This paper was supported by the Science and Engineering Research Council and by the University of Glasgow.

H. Ward, J. Hough, G. P. Newton, B. J. Meers, N. A. Robertson, S. Hoggan, G. A. Kerr, and J. B. Mangan are with the Department of Natural Philosophy, University of Glasgow G12 8QQ, Scotland.

R. W. P. Drever was with the Department of Natural Philosophy, University of Glasgow, Glasgow G12 8QQ, Scotland. He is now with the California Institute of Technology, Pasadena, CA 91125. measurements, the test masses can be suspended to give two mutually perpendicular baselines in which the gravitational wave signal will induce a differential displacement. The relative length of the two arms can be monitored by laser interferometry, and the optical phase changes can be enhanced by reflecting the light back and forth between a system of mirrors on the test masses, which may form optical delay lines [2] or resonant Fabry-Perot cavities [1].

\section{A Prototype 10-M Baseline Interferometer}

Experimental work on detectors using resonant optical cavities has been carried out at Glasgow University, Glasgow and California Institute of Technology Pasadena, CA [3]. The prototype interferometer being developed at Glasgow contains two high finesse $(\sim 600)$ resonant optical cavities with the cavity mirrors mounted on test masses which are hung as pendulums at three corners of a square of side $10 \mathrm{~m}$ (Fig. 1). The test masses and differential optical paths are contained in a vacuum system. The two cavities are illuminated by light from the same argon laser operating at $514 \mathrm{~nm}$ and both are kept close to resonance. Hence, small changes in the lengths of the cavities cause relatively large phase changes in the stored light, which can be sensed interferometrically.

The fundamental limit to sensitivity is photon noise in the detected light, and if cavities of finesse, $F$, are used in the system which is illuminated by light of intensity $I$, the differential displacement sensitivity can approach

$$
\mathrm{dl} \sim \sqrt{\left[h c \lambda / 8 \epsilon I F^{2}\right]} \quad \mathrm{m} / \sqrt{\mathrm{Hz}}
$$

where $\epsilon$ is the quantum efficiency of the photodiode and $\lambda$ is the wavelength of the light.

\section{A. General Description}

After some initial experiments [3] in which three mirror ring cavities were used to avoid optical feedback effects we have now adopted two mirror cavities which, having lower 


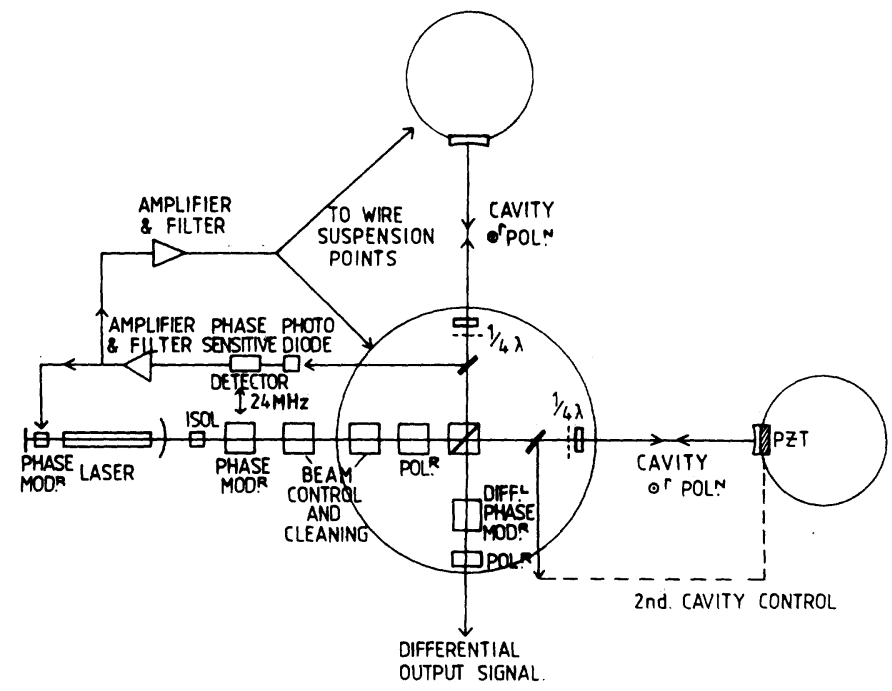

Fig. 1. Schematic diagram of prototype $10-\mathrm{m}$ baseline interferometer.

losses, can give higher finesse. As is shown in Fig. 1, an arrangement with a polarizing beamsplitter and quarter wave plates is used to separate input and output beams from the cavities, and to isolate the cavities from each other and from the laser. 10-m long cavities of high finesse (600) have a bandwidth $(25 \mathrm{kHz})$ considerably less than the fluctuations of the laser frequency and so a high degree of stabilization of the laser frequency with respect to the cavity resonances is required. In most of our work to date we have stabilized the laser to one of the $10-\mathrm{m}$ cavities and then stabilized the second cavity to the laser by means of a piezoelectrically driven mirror in the cavity. The feedback signal to this transducer gives a measure of the differential changes in length of the two baselines which could be induced by a gravitational wave.

\section{B. Stabilization of Laser and Cavities}

The stabilization schemes which are shown partly in Fig. 1 use our reflection RF sideband technique [4] .

Stabilization of the laser frequency is carried out as follows. The light from the laser is phase modulated by a transverse cut ADP crystal driven by $\sim 0.5 \mathrm{~W}$ of RF power at $24 \mathrm{MHz}$, a frequency considerably in excess of the transmission bandwidth of the cavity. The modulation produces RF sidebands of in dex $\sim 0.6$. The light which comes back from the front of the cavity can be considered to consist of two parts: one which has been simply reflected at the input mirror, and hence carries the phase modulation; and the other which leaks back out of the cavity and does not carry the phase modulation. The interference of these two components at the photodiode results in an amplitude modulated signal at $24 \mathrm{MHz}$, the level of the modulation being proportional to the frequency difference between the light from the laser and the light from the cavity. This signal after coherent demodulation, suitable amplification, and integral filtering, is applied to transducers to stabilize the frequency of the laser to that of the cavity. The servo amplifier/filters used are of the "bypass" design [4], [5] which allows minimum delay for high-frequency feedback signals. The overall transfer function of the system has a maximum rolloff of $\sim 18 \mathrm{~dB} /$ octave in the middle of its frequency range and a unity gain frequency of approximately $1 \mathrm{MHz}$. The transducers used are an intracavity electrooptic modulator over a range of $0-1 \mathrm{MHz}$ and a piezoelectrically driven mirror for low frequencies $(0-1 \mathrm{kHz})$.

It should be noted that the suspension of the test masses as pendulums allows large seismically excited changes in the cavity length at low frequencies. Part of the stabilizing signal for the laser is further filtered and fed to piezoelectric transducers driving the points of suspension of the pendulums to damp these motions. Measurements of the performance of the stabilization system show that a loop gain in excess of $10^{5}$ at $1 \mathrm{kHz}$ is achieved.

The same phase modulation technique is used to lock the resonant frequency of the second cavity to the frequency of the laser. Here again the light from the input mirror falls on a photodiode and is coherently detected. The signal is then amplified and filtered and fed to a piezoelectric transducer driving one of the mirrors in the cavity and, as for the first cavity, some of the feedback signal is used to damp the low frequency movement of the masses. The loop gain achieved $(\sim 10$ at $1 \mathrm{kHz}$ ) is much lower than that in the laser stabilization loop and is limited by the resonant frequency of the test mass carrying the piezoelectrically driven cavity mirror.

This second cavity can be regarded as a frequency analyzer for the laser light. The feedback signal is a measure of the difference between the resonant frequency of the cavity and the frequency of the laser, and it also gives a measure of the differential changes in length of the two baselines.

Control of orientation of the cavity mirrors to an angular stability of better than $10^{-5} \mathrm{rad}$ is achieved by applying signals from auxiliary optical systems using helium neon lasers to transducers which can move the supporting points of the wires suspending the test masses.

\section{Additional Features}

In our system, provision has been made for reducing fluctuations in the geometry-position, direction, diameter, and convergence-of the input laser beam.

Beam direction and position relative to the optical system on the center mass are monitored by a pair of quadrant photodiodes mounted on the mass itself. The outputs from these position-sensitive detectors control the orientation of four mirrors in the main laser beam path, by means of fast, piezoelectric transducers and slower, but wide-range, moving coil elements, so that the final beam is kept stable in position and direction. This system can reduce fluctuations in the beam position and direction by a significant factor. In particular the low-frequency servo has proved very valuable in maintaining the long-term alignment of the complete optical system and in simplifying adjustments of optical components such as electrooptic modulators and mode-matching lenses.

Further reduction in beam direction and position fluctuations, and reduction in beam diameter and convergence fluctuations, may be achieved by passing the beam through an auxiliary optical cavity mounted on the central mass and arranged to suppress all but one resonant mode [6]. This auxiliary cavity and the fast beam steering servo will be introduced after other noise sources have been investigated. 


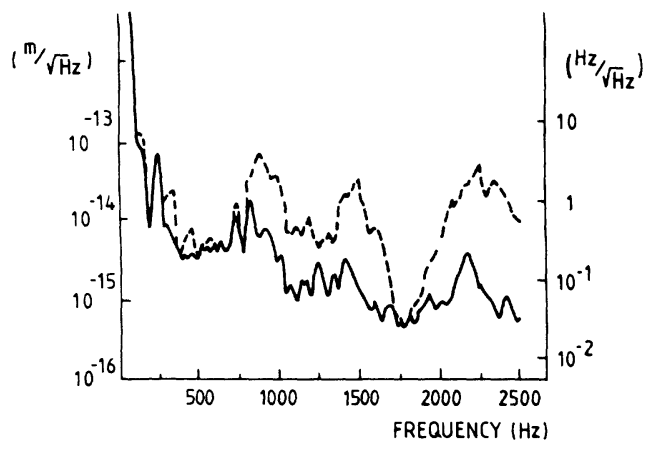

Fig. 2. Spectrum of noise in $10-\mathrm{m}$ interferometer (feedback signal to the second cavity). This can be interpreted as an upper limit to the spectral density of the frequency noise of the stabilized laser (righthand axis) or as the relative displacement noise spectrum. Dotted trace is with white noise excitation of the central test mass.

Provision is made for combining interferometrically the outputs from the two $10-\mathrm{m}$ cavities, a radio frequency phasemodulation technique being used to measure the phase difference between the two beams. This arrangement gives better fringe visibility and potentially better sensitivity than the use of separate detectors for each cavity and allows residual frequency noise in the laser to be balanced out.

Considerable attention has been paid to the mechanical design of the system. The end test masses are made of aluminum of high-quality factor; they are almost spherical in shape to keep their resonant frequencies high $(\sim 20 \mathrm{kHz})$ and so thermal noise from their internal modes should not contribute significantly to the noise level of the system at present. However, the middle test mass in necessarily of more complicated structure as it has to carry some optical modulators and beam steering optics as well as the beam cleaning cavity; this will be discussed further in the next section.

Good mechanical isolation of the test masses from external vibration in the system has been achieved by the introduction of mechanical filters of lead and rubber between the ground and the suspension points of the masses, and by removal of mechanically resonant components in the supporting structures.

\section{Performance of the 10-M Prototy pe System}

The overall performance of the system described above was as shown in Fig. 2. As noted before the finesse of each cavity was $~ 600$; the contrast of the fringes in reflection was $\sim 40$ percent from each cavity. Over a frequency band around 1800 $\mathrm{Hz}$ the sensitivity approached $3 \times 10^{-16} \mathrm{~m} / \sqrt{ } \mathrm{Hz}$, and was within a factor of approximately 4 of the limit set by the photon noise in the relatively small amount of light $(\sim 300 \mu \mathrm{W})$ used to control each of the laser and cavity stabilization systems. (Note that most of the light in the detector is intended to be used in the beam recombination part of the system, and the photon noise in this output will be much less significant.) However it did not quite reach photon noise in this frequency band. Evidence obtained by artificially exciting the mechanical resonances of the central mass suggested that much of the noise in the spectrum was due to excitation of these resonances. Fig. 2 shows a superposition of two system sensitivity spectra, with and without artificial excitation of the central mass. The similar structure in both, especially between 1.5 and $2 \mathrm{kHz}$, is clearly significant. When looked at over a wide frequency range the system did reach the photon noise limit at $17 \mathrm{kHz}$ and above; again it appeared that the excess noise below $17 \mathrm{kHz}$ was mainly due to mechanical resonances.

As was mentioned in the last section, the central test mass of the detector is of complicated construction and it is not surprising that it has resonances within the frequency band of interest whose effects are important. While it is difficult to simplify this mass, a way around the problem is to move each of the two central cavity mirrors onto its own separately suspended test mass of good quality factor and high internal resonant frequency. Further servo systems are required to stabilize the relative positions of these masses and the central mass and to control the orientations of the new masses. The possibility of using such an arrangement was envisaged from an early stage in the development of the detector, but it was felt to be more convenient to start operation of the system in the simplest way.

\section{Recent Development of the 10M INTERFEROMETER}

New central test masses and servo systems have now been added to our detector (Fig. 3), and initial tests indicate that this development has made a considerable improvement to the sensitivity spectrum as is shown in Fig. 4. This is essentially flat at a level of better than $1.5 \times 10^{-16} \mathrm{~m} / \sqrt{ } \mathrm{Hz}$ from approximately 1 to $2.5 \mathrm{kHz}$ and is now limited by the photon noise in the small amount of light used in the stabilization systems.

\section{Future Plans}

With the sensitivity in the kilohertz region of the spectrum now limited by photon noise it is appropriate to use the interfered output to improve the interferometer sensitivity. We have recently made some initial tests using the combined output and have observed $\sim 10 \mathrm{~dB}$ improvement in the system displacement noise level at high frequencies $(17 \mathrm{kHz})$. This is very encouraging and investigations at lower frequencies are about to be carried out. It is interesting to note that the performance of the subtraction system is affected by the relatively low gain in the servo stabilizing the second cavity to the laser frequency. This allows the effective sensitivity of the secondary cavity to residual frequency fluctuations in the laser to vary on short timescales, thereby impairing the accuracy with which these fluctuations are balanced out in the combined output. Improvements to the secondary cavity servo system are under way at present.

Further improvement in sensitivity will necessitate using mirrors of lower loss, and hence higher finesse in our cavities, and a considerable increase in the light level used to illuminate the system. Part of this increase can be obtained relatively easily by improving the quality of the beam handling optics, but a very important development for the future is to increase the level of single-mode frequency-stabilized light available from an argon ion laser.

Currently, a significant loss of light is due to the electrooptic modulator placed inside the laser cavity as the feedback trans- 


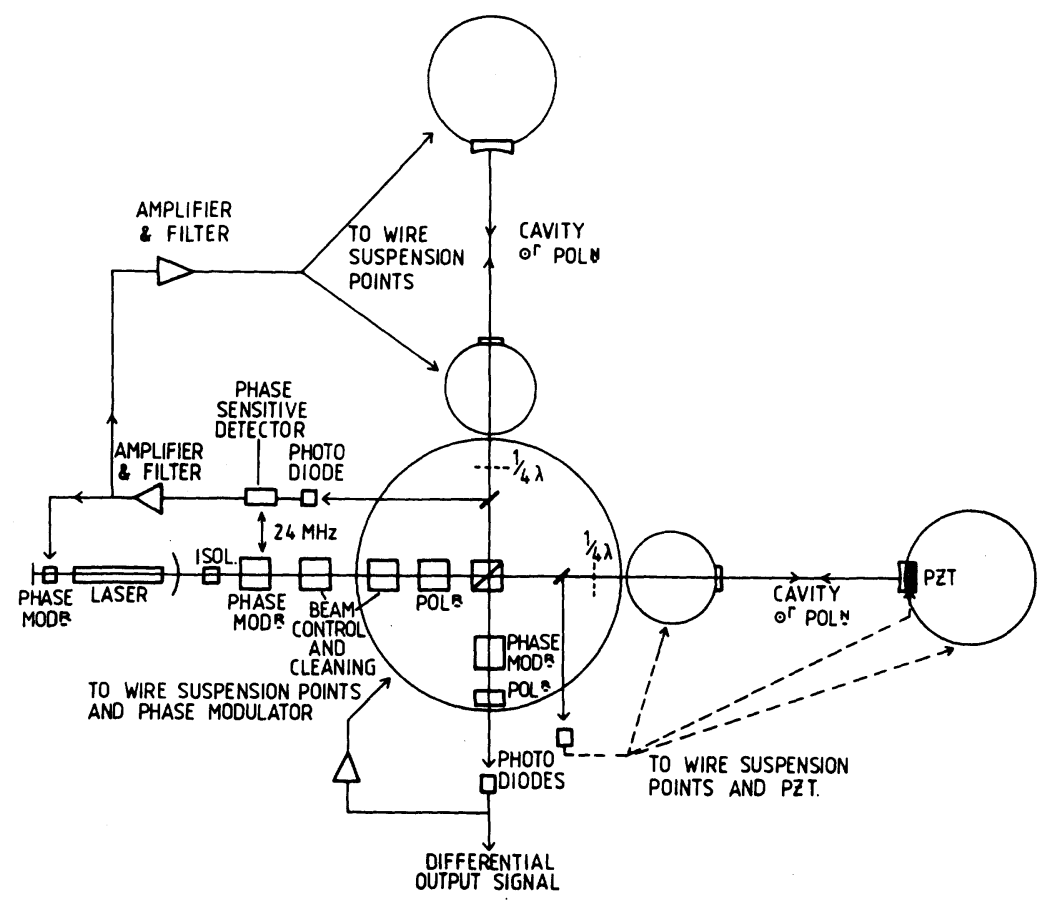

Fig. 3. Schematic diagram showing separately suspended test masses at the center of the interferometer.

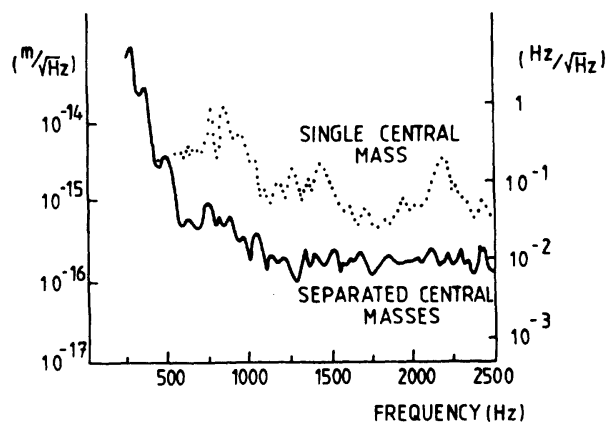

Fig. 4. Spectrum of noise in $10 \mathrm{~m}$ interferometer before and after incorporation of separate test masses at the center of the system.

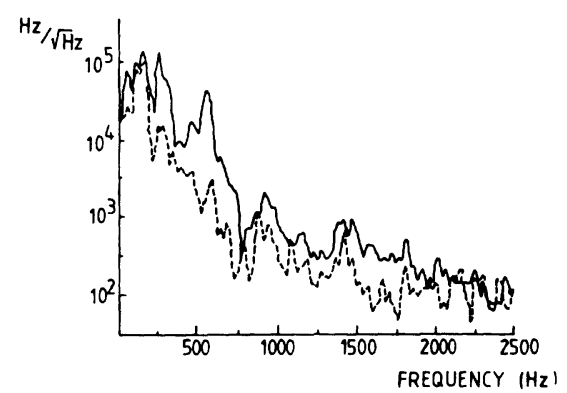

Fig. 5. Natural frequency noise of new laser tube. Solid curve-tube in standard resonator. Dotted curve-tube in separately built resonator.

ducer to stabilize its frequency. Such a device is necessary in most high-power lasers to provide a large enough gain-bandwidth product to suppress sufficiently their natural frequency fluctuations. We are tackling this problem at present using a commercially available laser tube of relatively low noise mounted in a specially built, mechanically damped resonator. The initial improvement in natural frequency noise when the separate resonator is used is shown in Fig. 5. Further, we are developing a method of stabilization which avoids a lossy intracavity modulator by utilizing a piezoelectrically driven laser

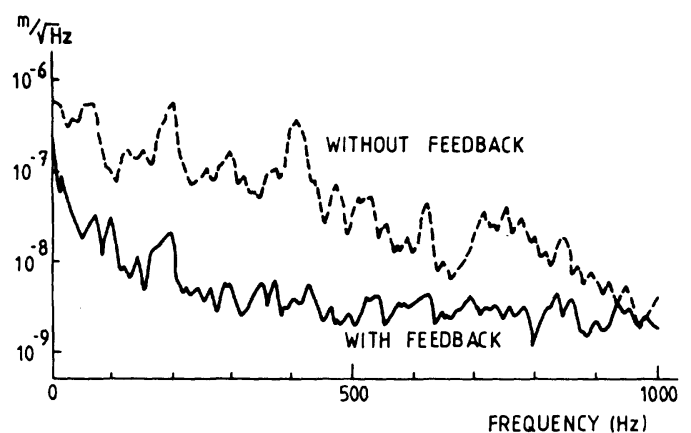

Fig. 6. Spectrum of positional fluctuations of the laser beam at a distance from the laser where the beam radius is $1 \mathrm{~mm}$-with and without the beam position stabilization system in operation.

cavity mirror in conjunction with a wideband phase/frequency adjusting element external to the laser cavity.

Lastly, it should be noted that in order to obtain the photon noise-limited performance with the eventual higher input power $(\sim 1 \mathrm{~W})$, it will be necessary to use the fast elements of the beam position stabilization system whose performance is shown in Fig. 6, and to introduce the mode-cleaning cavity into the beam line.

\section{CONCLUSION}

We have made significant advances in the development of laser interferometric displacement sensing of gravitational wave detectors, and propose to apply these techniques to an interferometer of $1-\mathrm{km}$ baseline which should allow detection of gravitational radiation from astrophysical sources.

\section{REFERENCES}

[1] R. W. P. Drever, in Gravitational Radiation, Proc. Nato Advanced Study Institute, Les Houches, N. Deruelle and T. Piran, Eds., Amsterdam, The Netherlands: North Holland, 1982, p. 321.

[2] H. Billing, W. Winkler, R. Schilling, A. Rudiger, K. Maischberger, 
and L. Schnupp, Quantum Optics, Experimental Gravity and Measurement Theory, P. Meystre and M. O. Scully, Eds. New York: Plenum, 1983, p. 525.

[3] J. Hough, R. W. P. Drever, A. J. Munley, S. A. Lee, R. Spero, S. E. Whitcomb, H. Ward, G. M. Ford, M. Hereld, N. A. Robertson, I. Kerr, J. R. Pugh, G. P. Newton, B. J. Meers, E. D. Brooks III, and Y. Gursel, Quantum Optics, Experimental Gravity and Measurement Theory, P. Meystre and M. O. Scully, Eds. New York: Plenum, 1983, p. 515.

[4] R. W. P. Drever, J. L. Hall, F. V. Kowalski, J. Hough, G. M. Ford,
A. J. Munley, and H. Ward, "Laser phase and frequency stabilization using an optical resonator," App. Phys. B, vol. 31, pp. 97$105,1983$.

[5] J. Helmcke, S. A. Lee, and J. L. Hall, "Dye laser spectrometer for ultra-high spectral resolution," App. Optics, vol. 21 , pp. 16861694, May 1982.

[6] A. Rudiger, R. Schilling, L. Schnupp, W. Winkler, H. Billing, and K. Maischberger, "A mode selector to suppress fluctuations in laser beam geometry," Opt. Acta, vol. 28, no. 5, pp. 641-658, 1981.

\title{
Precise Frequenccy Measurements of $\mathrm{CO}_{2} / \mathrm{OsO}_{4}$ and $\mathrm{HeNe} / \mathrm{CH}_{4}$-Stabilized Lasers
}

\author{
ANDRÉ CLAIRON, BRAHIM DAHMANI, ADRIAN FILIMON, AND J. RUTMAN
}

\begin{abstract}
New frequency measurements near $29 \mathrm{THz}$ have been performed with a resolution of about $\pm 1,710^{-12}(1 \sigma)$ on an $\mathrm{OsO}_{4}$ stabilized ${ }^{12} \mathrm{CO}_{2}{ }^{16} \mathrm{R}(10)$ laser. To this end, a new low-noise synthesis chain using two FIR optically pumped phase-locked lasers and a good spectral purity quartz oscillator at $1.06 \mathrm{GHz}$ have been developed. In order to allow comparisons with previous measurements, a new measurement of the $\mathrm{CH}_{4}$ stabilized $\mathrm{HeNe}$ laser was made using a ${ }^{12} \mathrm{CO}_{1}{ }^{16} \mathrm{O}_{2} \mathrm{R}(12) /$ $\mathrm{OsO}_{4}$ stabilized as a reference.
\end{abstract}

\section{INTRODUCTION}

$\mathrm{S}$ INCE the first absolute frequency measurement in the far infrared in 1967 [1], several laboratories have become involved with optical frequency measurements. These measurements rely on successive harmonic mixing using nonlinear devices. Various technical improvements have lead to recent measurement systems that are now more compact, [14], easier to use, and more precise than the earlier versions. Also, extension of frequency measurements into the visible has been performed [2].

Work in this field began at the Laboratoire Primaire du Temps et des Frequences (LPTF) Paris, France, in 1976-1977 and results have been presented at CPEM 1980 and 1982 [3], [4] In 1980, a first chain including a stable free-running $\mathrm{CH}_{3} \mathrm{OH}$ laser was described [3]. Precise frequency measurements were performed at a precision of $10^{-10}$ on $\mathrm{CO}_{2}\left(\mathrm{CO}_{2}\right)$ and $\mathrm{HeNe}\left(\mathrm{CH}_{4}\right)$ lasers. At this level the laser chain did not contribute significantly to the final uncertainty.

In 1982, a phase-locked FIR laser using the Stark effect was described [4]. A proposal was then made for a new chain including these devices as transfer oscillators and also a low-noise

Manuscript received August 22, 1984. This work was supported by the Bureau National de Metrologie, France.

A. Clairon and B. Dahmani are with the Laboratoire Primaire du Temps et des Frequences, 74014, Paris, France.

A. Filimon is with the Matra Radiocommunications Company. oscillator at $1.06 \mathrm{GHz}$. Since then, such a phase-locked chain has been realized and used in measuring the frequency of a ${ }^{12} \mathrm{CO}_{2}{ }^{16} \mathrm{O}_{2} \mathrm{R}(10)$ laser with improved frequency stabilization by saturated absorption in the $\mathrm{OsO}_{4}$ heavy molecule [5], also developed at LPTF in the meantime.

The present paper describes the $\mathrm{OsO}_{4}$ stabilized $\mathrm{CO}_{2}$ laser, the phase-locked chain including $\mathrm{HCOOH}(716 \mathrm{GHz})$ and $\mathrm{CH}_{3} \mathrm{OH}$ $(4.25 \mathrm{THz})$ lasers, and the experimental results obtained at the $10^{-12}$ level near $29 \mathrm{THz}$, and $3.10^{-11}$ level at $88 \mathrm{THz}$.

\section{II. $\mathrm{CO}_{2}$ LASER Stabilization AND FREQUENCY MEASUREMENT}

The reproducibility of $\mathrm{CO}_{2}$ lasers stabilized by saturated fluorescence in $\mathrm{CO}_{2}$ [6] is limited to about $10^{-10}$ by the attainable saturation peak width $(\simeq 1 \mathrm{MHz})$ and signal-to-noise $(\mathrm{S} / \mathrm{N})$ ratio. The heavy $\mathrm{OsO}_{4}$ molecule covers the $P(24)$ to $R(26)$ region of the $10-\mu \mathrm{m}$ branch of the $\mathrm{CO}_{2}$ laser and presents several interesting features: hyperfine structure is either absent or can be well resolved, owing to the high mass; and recoil splitting and second-order Doppler effect are small [7].

The $\mathrm{CO}_{2}$ laser frequency stabilization system is shown on Fig. 1. The $\mathrm{CO}_{2}$ sealed laser is designed for low-frequency noise and is shielded against acoustic noise; the beat note linewidth between such free-running lasers is less than $1 \mathrm{kHz}$. A few watts of output power made for easier laser isolation from feedback, since a few microwatts only are necessary for stabilization.

Saturated absorption of $\mathrm{OsO}_{4}$ is performed in a external Fabry-Perot cavity [8] in order to increase the saturation peak contrast and to obtain well-matched and clean Gaussian beams thus reducing frequency shifts associated with wavefront curvature. The 1.5-m long Fabry-Perot cavity comprises three invar rods and two $\mathrm{ZnSe}$ mirrors having $50 \mathrm{~m}$ radius of curvature and 2 percent transmission mounted on piezoelectric tranducers (PZT) to allow modulation and stabilization. The 